\title{
Posttransplantation Diabetes Mellitus after Renal Transplantation - A Brief Overview
}

\author{
Praveen Kumar E* \\ Department of Nephrology and Renal Transplantation, Asian Institute of \\ Nephrology and Urology, India \\ *Corresponding author: Dr. Etta Praveen Kumar, Department of Nephrology and \\ Renal Transplantation, Asian Institute of Nephrology and Urology, Hyderabad 500 \\ 082, Telangana, India, Email: drpraveen85@gmail.com
}

\section{Mini Review}

Volume 3 Special Issue 1

Received Date: March 01, 2019

Published Date: March 13, 2019

DOI: $10.23880 /$ ijtps-16000S1-004

\footnotetext{
Abstract

Posttransplantation diabetes mellitus (PTDM) occurs in a significant proportion of renal allograft recipients (RTRs) adversely affecting both patient and graft survival. It may be diagnosed at any time after transplantation, but highest incidence is seen in first one year. This article attempts to provide a brief overview of PTDM resulting after renal transplantation.
}

Keywords: Posttransplantation diabetes mellitus; New-onset diabetes mellitus after transplantation; Renal allograft; Hyperglycemia

\section{Introduction}

Posttransplantation diabetes mellitus (PTDM) or newonset diabetes mellitus after transplantation (NODAT) occurs in a significant number of renal transplant recipients (RTRs) and is associated with higher rates of cardiovascular disease (CVD) and infection, contributing to increased morbidity and mortality. It is characterized by both $\beta$-cell dysfunction and reduced insulin sensitivity. International consensus guidelines regarding PTDM were published in 2003 and updated in 2014, which prefers to use the term PTDM over NODAT, since some of these cases might represent unknown diabetes that was present before transplantation [1]. These guidelines use standard World Health Organization (WHO) and American Diabetes Association (ADA) criteria for the diagnosis [2].

PTDM may be diagnosed at any time after transplantation by any of the following:
- Symptoms of diabetes plus random plasma glucose $\geq 200 \mathrm{mg} / \mathrm{dL}$ (11.1 mmol/L)

- Fasting plasma glucose $\geq 126 \mathrm{mg} / \mathrm{dL}$ ( $7.0 \mathrm{mmol} / \mathrm{L})$

- Two-hour plasma glucose $\geq 200 \mathrm{mg} / \mathrm{dL}$ (11.1 mmol/L) during an oral glucose tolerance test (OGTT). The test should be performed as described by the WHO, using a glucose load containing the equivalent of $75 \mathrm{~g}$ anhydrous glucose dissolved in water.

The glycated hemoglobin (HbA1c) cannot be accurately interpreted within the first three months after transplantation and is not recommended for the diagnosis in early posttransplant period. After three months posttransplant, a HbA1c $\geq 6.5 \%$ can be used to diagnose diabetes by ADA criteria, with a HbA1c of 5.7-6.4\% consistent with prediabetic state.

The term "prediabetes," includes impaired fasting glucose and/or impaired glucose tolerance and is 


\section{International Journal of Transplantation \& Plastic Surgery}

diagnosed by a fasting plasma glucose between 100 and $125 \mathrm{mg} / \mathrm{dL}$ (5.6 and $6.9 \mathrm{mmol} / \mathrm{L}$ ) or a two-hour plasma glucose between 140 and $199 \mathrm{mg} / \mathrm{dL}$ (7.8 and 11.0 $\mathrm{mmol} / \mathrm{L}$ ) during an OGTT, respectively, according to ADA guidelines. The normal range of fasting plasma glucose differs according to ADA and WHO criteria; an abnormal fasting glucose is defined as $\geq 100 \mathrm{mg} / \mathrm{dL}(5.6 \mathrm{mmol} / \mathrm{L})$ by the ADA and $\geq 110 \mathrm{mg} / \mathrm{dl}(6.1 \mathrm{mmol} / \mathrm{L})$ by the WHO. The lower threshold advocated by the ADA is more sensitive in identifying patients at risk for PTDM [3]. The two-hour OGTT is more sensitive than the fasting blood glucose for detecting prediabetes. Postprandial hyperglycemia is the typical presentation of PTDM as opposed to elevated fasting blood glucose levels.

The incidence rates of PTDM are higher in the first one year after transplantation. The reported incidence rates are variable due to differences in the definitions used, time since transplantation, duration of follow-up, study population, immunosuppressive regimen, and other modifiable and non-modifiable risk factors. In the literature, the reported prevalences of PTDM varied from $16.75 \%$ to $54.5 \%$ [4-8]. Studies that use the current criteria for diagnosis suggest that up to one-third of nondiabetic RTRs develop persistently impaired glucose metabolism by six months posttransplantation, however the prevalence of PTDM appears to be decreased in recent years [9].

The risk factors for PTDM include increased age, obesity or other component of metabolic syndrome, African American race, Hispanic ethnicity, family history of diabetes, gestational diabetes, pretransplant impaired fasting glucose or impaired glucose tolerance, polycystic kidney disease, increased HLA mismatches, deceased donor allografts, history of acute rejection, hepatitis- $C$ (HCV) and cytomegalovirus (CMV) infections, use of immunosuppressive drugs like glucocorticoids, calcineurin inhibitors (CNIs) and mammalian target of rapamycin (mTOR) inhibitors [8]. The role of induction agents in causing PTDM is controversial. One study from North India concluded that relative risk of PTDM with basiliximab induction was 2.3 (95\% CI 1.4-3.9) compared to that of patients without induction [10].

Higher doses of glucocorticoids among RTRs have been associated with the development of PTDM. Steroid induced diabetes is characterized especially by increased postprandial blood glucose and reduced insulin sensitivity. Though glucocorticoid induced dysglycemia is dose dependent, complete steroid withdrawal has not been clearly shown to reduce the incidence of PTDM
$[11,12]$. Corticosteroids induce diabetes predominantly by promoting insulin resistance, and CNIs cause islet cell toxicity and may directly affect transcriptional regulation of insulin expression [13]. Hypomagnesemia induced by CNIs may also precipitate glucose intolerance. Among CNIs, tacrolimus is more diabetogenic than cyclosporine as shown in Diabetes Incidence after Renal Transplantation: Neoral C Monitoring Versus Tacrolimus (DIRECT) study [14]. In the Efficacy Limiting Toxicity Elimination (ELITE) study, higher rates of PTDM developed at one year in patients receiving low-dose tacrolimus $(8.4 \%)$ versus those receiving standard-dose cyclosporine (6\%), low-dose cyclosporine (4.2\%), and low-dose sirolimus (6.6\%) [15]. Few meta-analyses also showed higher incidence of PTDM in patients receiving tacrolimus compared to cyclosporine $[16,17]$. Sirolimus is also diabetogenic. In one study, conversion to sirolimus from tacrolimus or cyclosporine has been associated with a significant worsening rather than an improvement in insulin resistance [18]. Azathioprine and mycophenolate mofetil (MMF) do not induce PTDM. The glycemic effects of immunosuppressive drug combination regimens have been compared in few studies. The combination of either CNI with sirolimus may be more diabetogenic than the combination of a CNI with MMF [19]. Use of few drugs such as statins, angiotensin converting enzyme inhibitors (ACEI), angiotensin receptor blockers (ARB), and cotrimoxazole may be associated with a reduced risk of PTDM $[20,21]$.

In a meta-analysis HCV infected RTRs were at almost four times greater risk of developing PTDM compared with uninfected individuals [22]. Proposed mechanisms include HCV-induced islet cell dysfunction, insulin resistance, and abnormalities in glucose metabolism. HCV therapy prior to transplantation may reduce the incidence of PTDM. One larger retrospective study from North India which looked at long-term outcomes of HCV infected RTRs showed significantly high prevalence of PTDM in HCV positive group (39.6\%) as compared to HCV negative group (18.9\%) [23]. In this study all the HCV infected patients received therapy prior to transplantation. The role of CMV infection is controversial. In one study, asymptomatic CMV infection was associated with a fourfold increased risk of PTDM [24].

Pretransplant impaired glucose tolerance is a risk factor for the development of PTDM. In one study, 35\% of patients with PTDM had impaired glucose tolerance pretransplant [25]. Transient hyperglycemia is much more common in immediate posttransplant period and may itself is a risk factor for future development of PTDM. 


\section{International Journal of Transplantation \& Plastic Surgery}

The risk due to perioperative transient hyperglycemia is identified in few studies. In one study, $29 \%$ of patients with perioperative hyperglycemia developed PTDM within the first year after transplantation [26]. Another study showed that posttransplant hyperglycemia was associated with a fourfold increased risk of PTDM [27]. In the same study, PTDM was developed in $46.7 \%$ of patients with posttransplant hyperglycemia. Some researchers have defined PTDM as a long-term ( $>3$ months) requirement for glycemic control therapy to prevent overdiagnosis.

The development of PTDM has adverse effect upon both patient and graft survival, mainly by increased risk of CVD and infections. PTDM correlates with increased cardiovascular mortality, which is the most likely cause of poor long-term survival in these patients [28]. The mechanism by which PTDM may decrease allograft survival independent of increased mortality (deathcensored allograft loss) is not clear. De novo diabetic nephropathy in the allograft due to PTDM can also result in graft loss [29]. Another possibility is that efforts to decrease immunosuppressive therapy for glycemic control may increase rates of rejection. PTDM has also been associated with an increased risk for infections like urinary tract infection, pneumonia and CMV infections.

The management includes pretransplant evaluation, posttransplant monitoring, lifestyle modification, and anti-diabetic therapy. Pretransplant assessment should include proper history, evaluation for evidence of the metabolic syndrome and for other cardiovascular risk factors and PTDM risk assessment with fasting plasma glucose or OGTT. Posttransplant screening usually includes regular monitoring of blood glucose levels weekly during the first four weeks, then at three and six months posttransplant, and then yearly. HbA1c can be checked after three months posttransplant.

Nonpharmacologic therapy includes diet control, weight reduction and exercise. The control of other cardiovascular risk factors like hypertension and dyslipidemia are also important. The potential benefit of modification of immunosuppressive therapy must be weighed against the risk of rejection. Glucocorticoid dose may be decreased in patients with PTDM, but complete steroid withdrawal is not recommended due to increased risk of rejection. Switching to cyclosporine may be considered among patients in whom diabetes remains difficult to control on lower-dose tacrolimus. An openlabel randomized prospective pilot study from North India demonstrated a significant improvement in HbA1c in the cyclosporine conversion arm compared with tacrolimus continuation group among RTRs [30]. Conversion to sirolimus is not recommended as sirolimus may further worsen insulin resistance and glycemia.

Oral hypoglycemic agents either as monotherapy or combination therapy, or Insulin are required in most patients at least in the first few months after transplantation. Insulin is preferred during first few weeks after transplantation and one study suggested intensified early treatment with regimens that include basal insulin significantly reduce risk of PTDM after one year, presumably via insulin-mediated protection of $\beta$ cells [31]. The oral hypoglycemic agents like sulfonylureas, metformin (biguanide), meglitinides, dipeptidyl peptidase-4 (DPP-4) inhibitors or gliptins, and thiazolidinediones are the approved therapies for use in PTDM.

Among sulfonylureas, glipizide, gliclazide and glimepiride are preferred in patients with renal insufficiency. Since sulfonylureas may have potential toxicity in the setting of renal dysfunction, few prefer to use meglitinides, such as repaglinide. Gliptins or incretin mimetics are being widely used now even in RTRs. Gliptins do not cause hypoglycemia (due to glucose dependent action), do not induce weight gain and do not seem to interact with immunosuppressive drugs. Therefore it seems prudent to consider them as potential first-line oral drugs to treat PTDM. They inhibit DPP-4, the enzyme responsible for incretin degradation, which increases insulin synthesis and decreases glucagon levels. Sitagliptin, vildagliptin, repaglinide and nateglinide have been used among transplant recipients in short-term studies with documented safety and efficacy. Till date, only one double-blind, placebo controlled randomized trial has been performed in PTDM which concluded that vildagliptin is efficacious in RTRs [32]. Sitagliptin may prolong the QT interval, especially if used with cyclosporine. Linagliptin is cleared by extra-renal pathway and one Indian study evaluated its efficacy and safety in PTDM [33]. Incretin mimetics - exenatide, liraglutide and lixisenatide and sodium-glucose cotransporter 2 (SGLT2) inhibitors - canagliflozin, dapagliflozin, empagliflozin have not yet been validated for use in PTDM. Metformin can be initiated in patients with an estimated GFR (eGFR) as low as $45 \mathrm{ml} / \mathrm{min}$ and most cases of metformin induced lactic acidosis have been reported in combination with ACEI or ARB in patients with reduced GFR. Metformin is contraindicated in patients with an eGFR of $<30 \mathrm{~mL} / \mathrm{min}$ and not recommended if the eGFR is $<45 \mathrm{~mL} / \mathrm{min}$. 


\section{International Journal of Transplantation \& Plastic Surgery}

Thiazolidinediones may worsen bone loss, lead to weight gain, edema formation, anemia, congestive heart failure and predispose to CNI toxicity. They are generally used as a last resort of oral therapy. Alpha-glucosidase inhibitors may worsen gastrointestinal side effects of MMF.

\section{Conclusions}

PTDM is widely prevalent affecting approximately one-third of nondiabetic RTRs by six months posttransplantation. It adversely affects both patient and allograft survival. Glucocorticoids, CNIs (tacrolimus>cyclosporine) and sirolimus increase the risk of PTDM. The management includes pretransplant evaluation, posttransplant monitoring, lifestyle modification, and anti-diabetic therapy. Adjustment of immunosuppression therapy aimed at improving glucose tolerance must be weighed against the risk of rejection. Among patients with poorly controlled PTDM despite a reduction in tacrolimus dose, switching to cyclosporine may be considered. Sulfonylureas, metformin, meglitinides, gliptins, and insulin are the therapies commonly used in patients with PTDM.

\section{References}

1. Sharif A, Hecking M, de Vries AP, Porrini E, Hornum $M$, et al. (2014) Proceedings from an international consensus meeting on posttransplantation diabetes mellitus: recommendations and future directions. Am J Transplant 14(9): 1992-2000.

2. American Diabetes Association (2011) Standards of medical care in diabetes-2011. Diabetes Care 34(1): S11-S61.

3. Valderhaug TG, Jenssen T, Hartmann A, Midtvedt K, Holdaas H, et al. (2009) Fasting plasma glucose and glycosylated hemoglobin in the screening for diabetes mellitus after renal transplantation. Transplan 88(3): 429-434.

4. Prakash J, Rathore SS, Singh TB, Choudhury TA, Prabhakar, et al. (2012) New onset diabetes after transplantation (NODAT): analysis of pre-transplant risk factors in renal allograft recipients. Indian J Transplant 6(3):77-82.

5. Sharma A, Minz M, Singh S (2008) Incidence of glucose metabolic abnormalities in Indian living renal allograft recipients on tacrolimus-based triple drug immunosuppression. Transplant Proc 40(7): 24142415.
6. Sanyal D, Das P, Gupta S, Bhattacharjee K (2017) Evaluation of Pre-Transplant Risk Factors as Independent Predictors on the New Onset of Diabetes after Renal Transplants (NODAT). J Endocrinol Thyroid Res 2(1): 555-576.

7. Bora GS, Guleria S, Reddy VS, Tandon N, Gupta N, et al. (2010) Risk factors for the development of new-onset diabetes mellitus in a living related renal transplant program. Transplant Proc 42(10): 4072-4073.

8. Jayam J, Balakrishnan MA, Srinivasaprasad ND, Sujit S, Fernando ME (2018) Prevalence and risk factors for posttransplant diabetes mellitus data from government tertiary care centre. Indian J Transplant 12(2): 119-124.

9. Matas AJ, Smith JM, Skeans MA, Thompson B, Gustafson SK, et al. (2015) OPTN/SRTR 2013 Annual Data Report: kidney. Am J Transplant 15 (2): 1-34.

10. Prasad N, Gurjer D, Bhadauria D, Gupta A, Srivastava A, et al. (2014) Is basiliximab induction, a novel risk factor for new onset diabetes after transplantation for living donor renal allograft recipients? Nephrology (Carlton) 19(4): 244-250.

11. Pirsch JD, Henning AK, First MR, Fitzsimmons W, Gaber AO, et al. (2015) New-Onset Diabetes After Transplantation: Results From a Double-Blind Early Corticosteroid Withdrawal Trial. Am J Transplant 15(7): 1982-1990.

12. Woodle ES, First MR, Pirsch J, Shihab F, Gaber AO, et al. (2008) A prospective, randomized, double-blind, placebo-controlled multicenter trial comparing early (7 day) corticosteroid cessation versus long-term, low-dose corticosteroid therapy. Ann Surg 248: 564577.

13. Burroughs TE, Lentine KL, Takemoto SK, Swindle J, Machnicki G, et al. (2007) Influence of early posttransplantation prednisone and calcineurin inhibitor dosages on the incidence of new-onset diabetes. Clin J Am Soc Nephrol 2(3): 517-523.

14. Vincenti F, Friman S, Scheuermann E, Rostaing L, Jenssen T, et al. (2007) Results of an international, randomized trial comparing glucose metabolism disorders and outcome with cyclosporine versus tacrolimus. Am J Transplant 7(6): 1506-1514.

15. Ekberg H, Tedesco-Silva H, Demirbas A, Vítko S, Nashan B, et al. (2007) Reduced exposure to 


\section{International Journal of Transplantation \& Plastic Surgery}

calcineurin inhibitors in renal transplantation. N Engl J Med 357: 2562-2575.

16. Heisel O, Heisel R, Balshaw R, Keown P (2004) New onset diabetes mellitus in patients receiving calcineurin inhibitors: a systematic review and metaanalysis. Am J Transplant 4(4): 583-595.

17. Webster AC, Woodroffe RC, Taylor RS, Chapman JR, Craig JC (2005) Tacrolimus versus ciclosporin as primary immunosuppression for kidney transplant recipients: meta-analysis and meta-regression of randomized trial data. BMJ 331(7520): 810.

18. Johnston O, Rose CL, Webster AC, Gill JS (2008) Sirolimus is associated with new-onset diabetes in kidney transplant recipients. J Am Soc Nephrol 19(7): 1411-1418.

19. Luan FL, Steffick DE, Ojo AO (2011) New-onset diabetes mellitus in kidney transplant recipients discharged on steroid-free immunosuppression. Transplant 91(3): 334-341.

20. Prasad GV, Kim SJ, Huang M, Nash MM, Zaltzman JS, et al. (2004) Reduced incidence of new-onset diabetes mellitus after renal transplantation with 3-hydroxy-3methylglutaryl-coenzyme a reductase inhibitors (statins). Am J Transplant 4(11): 1897-903.

21. Strevel EL, Kuper A, Gold WL (2006) Severe and protracted hypoglycaemia associated with cotrimoxazole use. Lancet Infect Dis 6(3): 178-182.

22. Fabrizi F, Martin P, Dixit V, Bunnapradist S, Kanwal F, et al. (2005) Post-transplant diabetes mellitus and HCV seropositive status after renal transplantation: meta-analysis of clinical studies. Am J Transplant 5(10): 2433-2440.

23. Prasad N, Etta PK, Jaiswal A, Sharma RK, Bhadauria D, et al. (2017) Long-term outcomes of hepatitis C virus infected renal allograft recipients. Indian J Transplant 11(2): 35-41.

24. Hjelmesaeth J, Sagedal S, Hartmann A, Rollag H, Egeland T, et al. (2004) Asymptomatic cytomegalovirus infection is associated with increased risk of new-onset diabetes mellitus and impaired insulin release after renal transplantation. Diabetologia 47(9): 1550-1556.

25. Caillard S, Eprinchard L, Perrin P, Braun L, Heibel F, et al. (2011) Incidence and risk factors of glucose metabolism disorders in kidney transplant recipients: role of systematic screening by oral glucose tolerance test. Transplant 91: 757-764.

26. Chakkera HA, Knowler WC, Devarapalli Y, Weil EJ, Heilman RL, Dueck A, et al. (2010) Relationship between inpatient hyperglycemia and insulin treatment after kidney transplantation and future new onset diabetes mellitus. Clin J Am Soc Nephrol 5(9): 1669-1675.

27. Memon SS, Tandon N, Mahajan S, Bansal VK, Krishna A, et al. (2017) The prevalence of new onset diabetes mellitus after renal transplantation in patients with immediate posttransplant hyperglycemia in a tertiary care centre. Indian J Endocr Metab 21(6): 871-875.

28. Fernández-Fresnedo G, Escallada R, de Francisco AL, Rodrigo E, Zubimendi JA, et al. (2003) Posttransplant diabetes is a cardiovascular risk factor in renal transplant patients. Transplant Proc 35(2): 700.

29. Owda AK, Abdallah AH, Haleem A, Hawas FA, Mousa D, et al. (1999) De novo diabetes mellitus in kidney allografts: nodular sclerosis and diffuse glomerulosclerosis leading to graft failure. Nephrol Dial Transplant 14(8): 2004-2007.

30. Rathi M, Rajkumar V, Rao N, Sharma A, Kumar S, et al. (2015) Conversion from tacrolimus to cyclosporine in patients with new-onset diabetes after renal transplant: an open-label randomized prospective pilot study. Transplant Proc 47(4): 115811-115861.

31. Hecking M, Haidinger M, Doller D, Werzowa J, Tura A, et al. (2012) Early basal insulin therapy decreases new-onset diabetes after renal transplantation. J Am Soc Nephrol 23(4): 739-749.

32. Haidinger $M$, Werzowa J, Hecking $M$, Antlanger $M$, Stemer G, et al. (2014) Efficacy and safety of vildagliptin in new-onset diabetes after kidney transplantation--a randomized, double-blind, placebo-controlled trial. Am J Transplant 14(1): 115123.

33. Sanyal D, Gupta S, Das P (2013) A retrospective study evaluating efficacy and safety of linagliptin in treatment of NODAT (in renal transplant recipients) in a real world setting. Indian J Endocrinol Metab 17(1): S203-S205. 